\title{
Social class and gender patterning of insomnia symptoms and psychiatric distress: a 20-year prospective cohort study
}

\author{
Michael J Green ${ }^{1 *}$, Colin A Espie ${ }^{2}$ and Michael Benzeval ${ }^{1,3}$
}

\begin{abstract}
Background: Psychiatric distress and insomnia symptoms exhibit similar patterning by gender and socioeconomic position. Prospective evidence indicates a bi-directional relationship between psychiatric distress and insomnia symptoms so similarities in social patterning may not be coincidental. Treatment for insomnia can also improve distress outcomes. We investigate the extent to which the prospective patterning of distress over 20 years is associated with insomnia symptoms over that period.

Methods: 999 respondents to the Twenty-07 Study had been followed for 20 years from approximately ages 36-57 (73.2\% of the living baseline sample). Psychiatric distress was measured using the GHQ-12 at baseline and at 20-year follow-up. Gender and social class were ascertained at baseline. Insomnia symptoms were self-reported approximately every five years. Latent class analysis was used to classify patterns of insomnia symptoms over the 20 years. Structural Equation Models were used to assess how much of the social patterning of distress was associated with insomnia symptoms. Missing data was addressed with a combination of multiple-imputation and weighting.
\end{abstract}

Results: Patterns of insomnia symptoms over 20 years were classified as either healthy, episodic, developing or chronic. Respondents from a manual social class were more likely to experience episodic, developing or chronic patterns than those from non-manual occupations but this was mostly explained by baseline psychiatric distress. People in manual occupations experiencing psychiatric distress however were particularly likely to experience chronic patterns of insomnia symptoms. Women were more likely to experience a developing pattern than men, independent of baseline distress. Psychiatric distress was more persistent over the 20 years for those in manual social classes and this effect disappeared when adjusting for insomnia symptoms. Irrespective of baseline symptoms, women, and especially those in a manual social class, were more likely than men to experience distress at age 57. This overall association for gender, but not the interaction with social class, was explained after adjusting for insomnia symptoms. Sensitivity analyses supported these findings.

Conclusions: Gender and socioeconomic inequalities in psychiatric distress are strongly associated with inequalities in insomnia symptoms. Treatment of insomnia or measures to promote healthier sleeping may therefore help alleviate inequalities in psychiatric distress.

Keywords: Insomnia, Psychiatric distress, Longitudinal, Socioeconomic position, Gender

\footnotetext{
* Correspondence: michael.green@glasgow.ac.uk

${ }^{1} \mathrm{MRC/CSO}$ Social and Public Health Sciences Unit, University of Glasgow,

4 Lilybank Gardens, Glasgow, G12 8RZ, UK

Full list of author information is available at the end of the article
} 


\section{Background}

Anxiety and depression symptoms (or psychiatric distress) are more common for those in a disadvantaged socioeconomic position (SEP) [1-4] and inequalities widen with increasing age [5-8]. Gender inequalities, with higher levels of psychiatric distress among adult women, are also a common finding $[9,10]$, and these too widen with age $[11,12]$. Gender differences are partially but not completely explained by female socioeconomic disadvantages [11]. Additionally, psychiatric distress predicts later insomnia symptoms (defined here as trouble initiating or maintaining sleep) $[13,14]$, and, vice versa, insomnia predicts later psychiatric distress [14-18], even with adjustment for baseline or historic symptoms. This bidirectional evidence is suggestive of a positive feedback loop where each problem aggravates the other. Indeed, insomnia symptoms exhibit similar social patterning. A female propensity for insomnia symptoms, especially at older ages, has been established in a metaanalysis [19], and cross-sectional studies on population samples indicate that insomnia symptoms are more commonly experienced by those in a disadvantaged SEP [20,21]. Gender differences in insomnia symptoms are attenuated but not fully explained with adjustment for socioeconomic position [20,22,23]. Longitudinal evidence on insomnia is sparse but indicates that women have a propensity to develop insomnia symptoms in middle age and that those in a disadvantaged SEP are more likely to experience chronic, persistent insomnia symptoms than those more affluent [23]. The mutual association between insomnia symptoms and psychiatric distress may mean the similarities in patterning are not coincidental.

Since insomnia symptoms can come within the range of symptoms that indicate psychiatric distress, it is tempting perhaps to dismiss the social patterning of insomnia symptoms as an expression of inequalities in psychiatric distress. This may be true, but insomnia symptoms can lead to greater distress as well as vice versa. Indeed, trials have shown improved depression outcomes where concurrent insomnia is treated [24,25] and some have even called for trials of insomnia treatment to prevent depression $[17,26]$. If inequalities in psychiatric distress are even partially mediated by inequalities in insomnia symptoms then insomnia treatment might have the added benefit of reducing inequalities in psychiatric distress. With this in mind we investigated the extent to which long-term sleep patterns might explain subsequent inequalities in psychiatric distress, taking earlier distress into account. We focus on symptoms in mid-life as this is the life-stage where there is the most widening of socioeconomic inequalities in psychiatric distress [8], and where women are particularly likely to develop insomnia symptoms [23].

\section{Methods}

\section{Sample and measures}

The Twenty-07 Study [27] has followed people in three age cohorts - born around 1932, 1952, and 1972 - for 20 years. It has two samples: the regional sample, a twostage stratified random sample of people living in an area of the West of Scotland centred on Glasgow (previously known as the Central Clydeside Conurbation), and the localities sample of people from two areas of the city of Glasgow. Baseline interviews were conducted in 1987/ 88 and there have been four follow-ups (1990/2; 1995/7; $2000 / 4 ; 2007 / 8)$. Ethical approval was gained for each wave from the NHS and/or Glasgow University Ethics Committees. The 1950s cohort aged from approximately 36 to 57 years during the study period, with a baseline sample size of 1,444 (response rate was $88.9 \%$ ). The achieved sample has been shown to be representative of the general population of the sampled area [28]. This paper focuses on a sub-set of respondents from this cohort who participated in both the baseline and the final interview in $2007 / 08(n=999 ; 73.2 \%$ of the living baseline sample).

Insomnia symptoms (latency and maintenance) were self-reported by the study respondents at each wave. For latency, in the first three interviews respondents were asked, "How often do you have trouble getting to sleep?" and in interviews four and five, "During the past month how often have you had trouble sleeping because you cannot get to sleep within 30 minutes?". For maintenance, in the first three interviews respondents were asked "How often are you bothered by waking earlier than you would like to, or by waking up in the middle of the night?" and in interviews four and five: "During the past month how often have you had trouble sleeping because you wake up in the middle of the night or early morning?". Responses were coded to indicate problems occurring at least weekly. A latent class variable was derived from the responses at all five interviews which categorised individual longitudinal patterns of insomnia symptoms into the four groups depicted in Figure 1 (for further details, including a consideration of the change in question wording, see Green et al. 2012) [23]. The four groups included: a Healthy pattern with low levels of symptoms across the 20 years; a pattern with episodic problems maintaining sleep, but relatively few problems initiating sleep (Episodic Maintenance); a pattern where insomnia symptoms were not present initially but developed later (Developing); and a chronic pattern with a high likelihood of problems both maintaining and initiating sleep across the 20 years (Chronic Mixed). This latent class variable constitutes the measure of insomnia symptoms used in this study and represents an individual's pattern of symptoms across the 20 year study period rather than at any single point in time. Classification of individuals into classes 


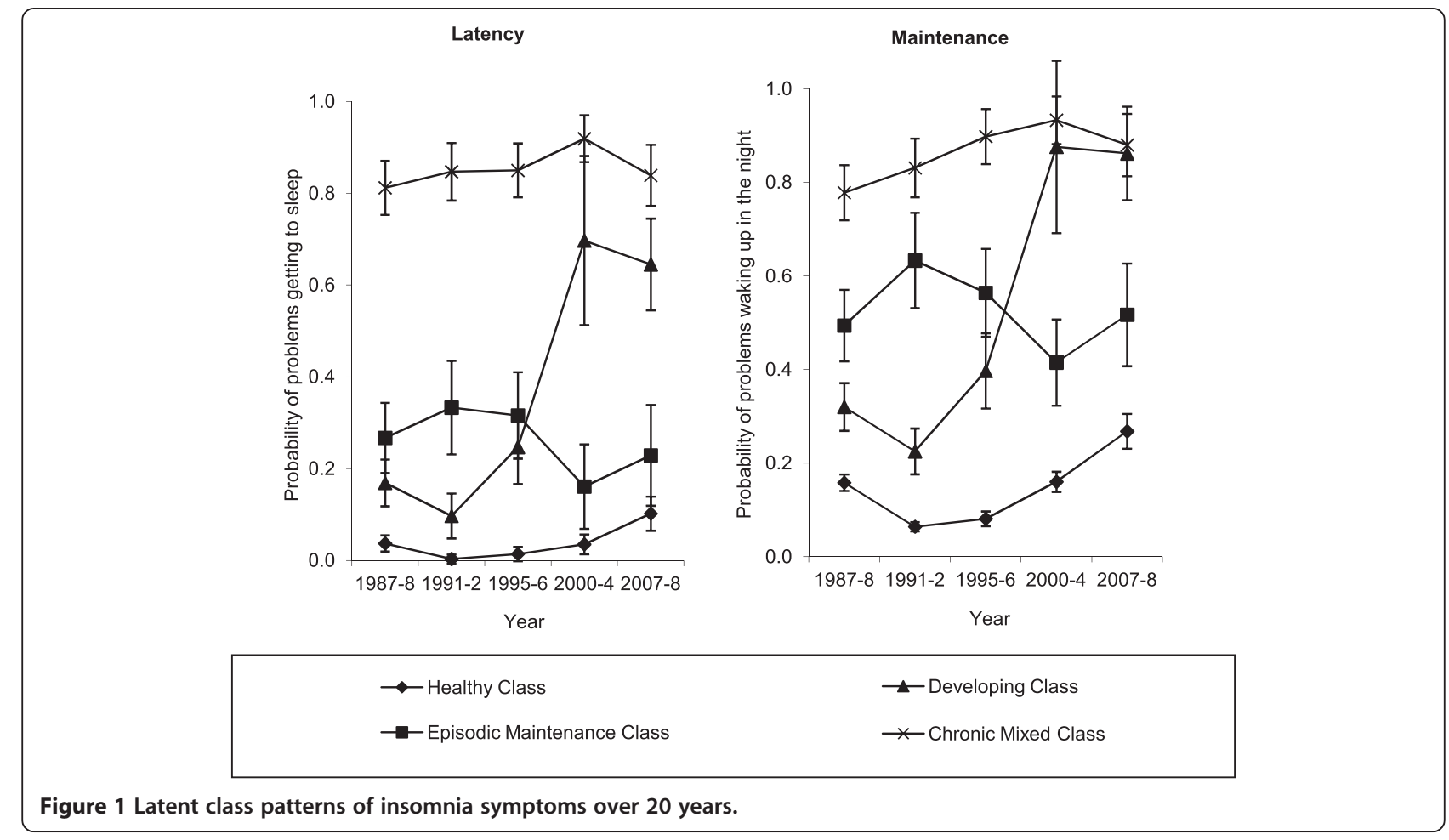

was reasonably definitive (entropy statistic: 0.68 ), but not certain.

Psychiatric distress was measured at baseline and at the final interview using the 12-item General Health Questionnaire (GHQ) [29]. Scores of 2 or more were coded as indicating probable psychiatric morbidity. Gender was coded 0 for males and 1 for females. SEP was measured with baseline household occupational class, coded according to the Registrar General's 1980 classification [30], using the higher status occupation from couple households. If neither the respondent nor their partner had a current occupation then the most recent previous occupations were used. This was dichotomised with 0 for non-manual (I through to III non-manual) and 1 for manual (III manual through to V). Medications were self-reported and then assigned British National Formulary codes by medically-trained coders.

\section{Statistical analyses}

Figure 2 illustrates the structural equation model used. A structural equation model can be thought of as a combination of measurement models, in which latent variables are defined, and a structural model describing relationships between those latent variables and other observed variables. The measurement model used here is the latent class variable representing longitudinal patterns of insomnia symptoms. This is estimated jointly with the wider structural equation model, in order to account for the fact that latent class membership is uncertain [31].
The structural part of the model relates this latent class variable to other observed variables, proceeding through four main stages. Initially, gender and baseline social class are used as predictors of latent class membership (model A). This establishes the gender and social class patterning of the latent classes, replicating our previous findings [23]. Adjustment is then made for baseline psychiatric distress (model B) in order to see how much of the gender and social class patterning of insomnia symptoms can be accounted for by initial distress.

Prospective associations between gender and baseline social class and psychiatric distress at age 57 are then estimated with adjustment for baseline psychiatric distress (model C). Finally, psychiatric distress at age 57 is additionally adjusted for latent class membership (model D), in order to see how much of the prospective associations with gender and social class are mediated via long-term sleeping patterns. Interactions between gender, social class and psychiatric distress were tested at all stages and were retained if they were significant at the $\mathrm{P}<0.05$ level at any stage. Adding variables to a latent class model can potentially alter class membership assignments [31] so parameters were fixed to enhance stability across model stages. Class assignments were generally more than 95\% stable across different models, and the few who did switch classes between model stages tended to be those who were uncertainly assigned anyway (i.e. their responses didn't fit well with any of the four patterns).

Analyses were conducted in Mplus version 7 [32], using maximum likelihood estimation with robust standard 


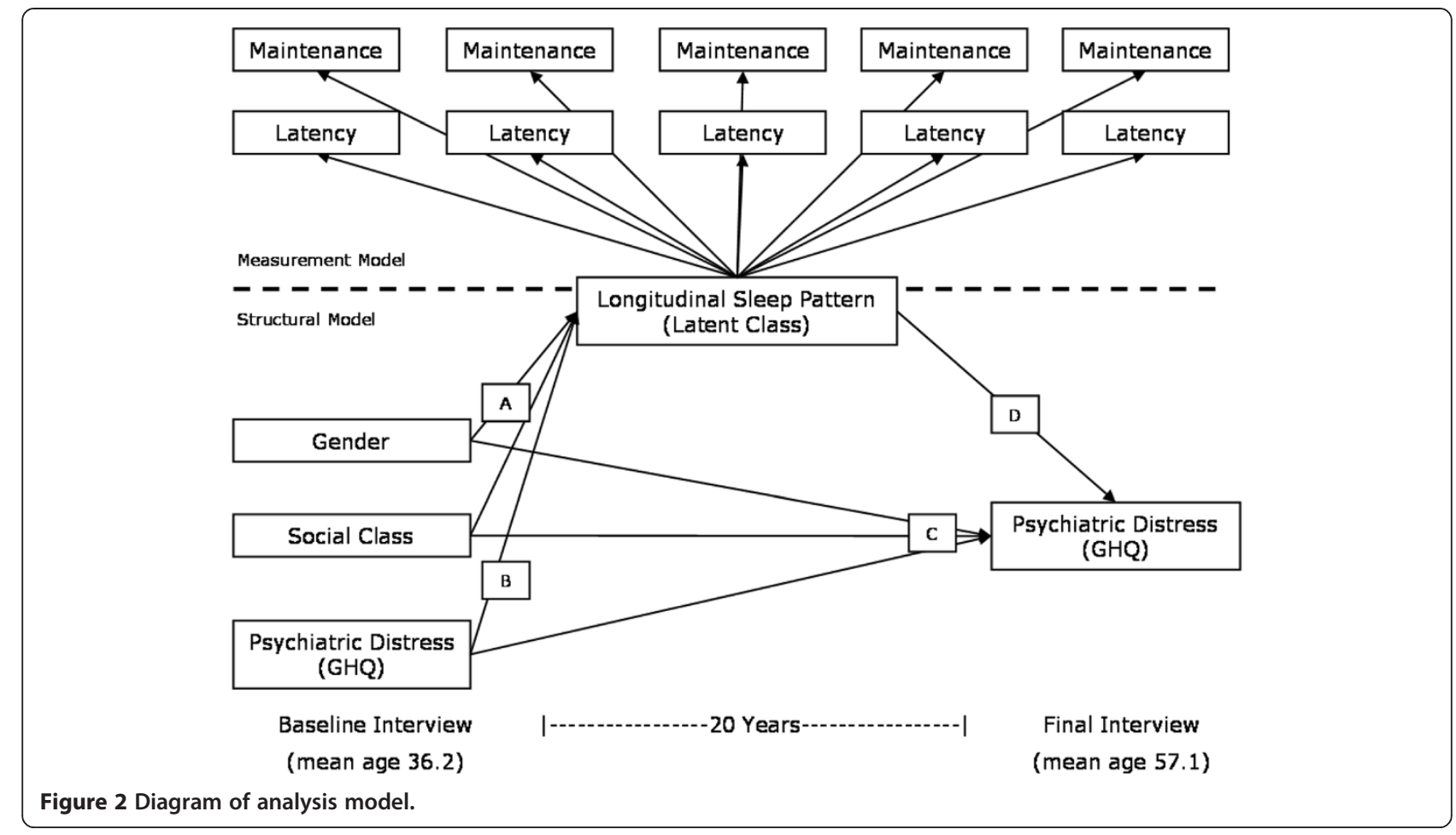

errors to account for non-normality of data and clustered sampling. Results are presented as odds ratios (OR) with 95\% confidence intervals $(95 \% \mathrm{CI})$. Considering attrition, the analysis sample was weighted to the living baseline sample [33]. However, of the 999 who participated in the fifth interview, only 859 (86\%) had full data for all the variables in the structural part of the model. Multiple imputation with an unrestricted model [34] of all the analysis variables (including the weights) was therefore used to estimate missing values for the structural part of the model. It was not necessary to impute data for the measurement part of the model as respondents could still be assigned to latent classes based on their observed responses. Additionally, data on GHQ scores and anti-depressant medication from intervening interviews were included where available to strengthen the imputation model [35]. Fifteen imputed datasets were created and analysis results were averaged across these datasets [36].

Three further sensitivity analyses were conducted, essentially repeating the main analyses (including the imputation of missing values) with different definitions of psychiatric distress. Two further binary definitions were used. First, as one of the GHQ items ('Have you recently lost much sleep over worry?') relates to sleeping difficulties the analysis was repeated with this item excluded from the calculation of the GHQ scores. This tests whether observed associations are the result of measurement overlap. Second, as some respondents might have had low GHQ scores because they were taking anti-depressant medication to manage their symptoms the analysis was repeated with psychiatric distress indicated by either a high GHQ score (2+), or reporting taking anti-depressant medication. Finally, the analyses were also repeated using the continuous GHQ scores, to see if results differed with greater sensitivity to the severity of psychiatric distress being experienced.

\section{Results}

Descriptive characteristics of the sample (before weighting and imputation) are displayed in Table 1. Psychiatric distress was present in a little more than a quarter of the sample at both ages 36 and 57. The Healthy sleep pattern was most common, followed by the Developing and Episodic Maintenance patterns, whilst Chronic Mixed was least common.

Table 2 displays the results from the first two stages. In model A, as found previously [23], relative to those with Healthy sleep patterns, women were more likely to be in the Developing class than men. In reference to the Healthy class, those from manual social classes were more likely to be in any of the symptomatic sleep classes than those from non-manual occupations, though this relationship was weak ( $\mathrm{p}=0.097$ ) for the Episodic Maintenance class, and strongest for the Chronic Mixed class. When baseline psychiatric distress was adjusted for in model B, the associations between sleep classes and gender were largely unaffected but social class was no longer significantly associated with membership in the Episodic 
Table 1 Descriptive data for the analysis variables

\begin{tabular}{lcc}
\hline Sample characteristics $(\mathbf{n}=\mathbf{9 9 9})$ & N (\%) \\
\hline Gender & Male & $457(45.7)$ \\
Social class at age 36 & Female & $542(54.3)$ \\
& Non-manual & $686(68.7)$ \\
& Manual & $304(30.4)$ \\
GHQ (2+) at age 36 & Missing & $9(0.9)$ \\
& No & $593(59.4)$ \\
GHQ (2+) at age 57 & Yes & $303(30.3)$ \\
& Missing & $103(10.3)$ \\
Most likely sleep class & No & $706(70.7)$ \\
& Yes & $255(25.5)$ \\
& Missing & $38(3.8)$ \\
& Healthy & $464(46.4)$ \\
& Episodic maintenance & $164(16.4)$ \\
& Developing & $250(25.0)$ \\
& Chronic Mixed & $112(11.2)$ \\
& Missing & $9(0.9)$ \\
\hline
\end{tabular}

Maintenance or Chronic Mixed classes and the association weakened for the Developing class $(\mathrm{p}=0.051)$. Respondents with baseline psychiatric distress were generally more likely to be members of symptomatic sleep classes than to be healthy sleepers. However, social class was still important for sleep as an interaction was found whereby those in a manual social class with high baseline GHQ scores were especially likely to be in the Chronic Mixed class (rather than the Healthy class). All three sensitivity analyses replicated these findings (see Additional file 1).

The results from models predicting psychiatric distress at age 57 are displayed in Table 3. In model C, without adjustment for sleep class membership, women and those with high baseline GHQ scores were more likely to have high GHQ scores at age 57. Both of these effects were stronger for those in manual social classes relative to their non-manual counter-parts, though the gender by social class interaction was statistically weak $(\mathrm{p}=0.072)$. Model $\mathrm{D}$ adjusted for sleep class membership. All of the symptomatic sleep classes, but especially the Chronic Mixed class, were associated with higher odds of psychiatric distress at age 57 than in the Healthy sleep class. The gender and baseline GHQ effects were attenuated, but only the gender difference became non-significant. The interaction between social class and baseline GHQ was also attenuated into non-significance, but the interaction between social class and gender was actually stronger with adjustment for insomnia symptoms, passing the $\mathrm{p}<0.05$ level.

The sensitivity analyses for models $\mathrm{C}$ and $\mathrm{D}$ produced broadly consistent findings (details in Additional file 1). The Episodic Maintenance, Developing, and Chronic Mixed classes (relative to the Healthy class) were associated respectively with mean differences of 1.04, 1.36 and 2.16 GHQ points at age 57 (standard errors were $0.30,0.24$ and 0.41 ), adjusting for the other variables in the model (including baseline GHQ scores).

Table 2 Odds ratios for sleep class membership with and without adjustment for baseline psychiatric distress

\begin{tabular}{|c|c|c|c|c|}
\hline & \multicolumn{2}{|c|}{ Model A: Predicting sleep } & \multicolumn{2}{|c|}{ Model B: Predicting sleep with adjustment for psychiatric distress } \\
\hline & OR & $95 \% \mathrm{Cl}$ & OR & $95 \% \mathrm{Cl}$ \\
\hline \multicolumn{5}{|c|}{ Episodic maintenance class (ref: Healthy) } \\
\hline Female gender & 0.85 & $(0.49-1.47)$ & 0.81 & $(0.45-1.46)$ \\
\hline Manual social class & $1.60^{*}$ & $(0.92-2.78)$ & 1.55 & $(0.65-3.69)$ \\
\hline GHQ $(2+)$ at age 36 & & & $3.57^{* *}$ & $(1.82-7.02)$ \\
\hline Social class and GHQ interaction & & & 1.26 & $(0.31-5.10)$ \\
\hline \multicolumn{5}{|l|}{ Developing class (ref: Healthy) } \\
\hline Female gender & $4.20^{* *}$ & $(2.68-6.60)$ & $4.02^{* *}$ & $(2.52-6.42)$ \\
\hline Manual social class & $1.86^{* *}$ & $(1.27-2.72)$ & $1.73^{*}$ & $(1.00-2.99)$ \\
\hline GHQ (2+) at age 36 & & & $2.32^{* *}$ & $(1.26-4.28)$ \\
\hline Social class and GHQ interaction & & & 1.54 & $(0.40-5.97)$ \\
\hline \multicolumn{5}{|l|}{ Chronic mixed class (ref: Healthy) } \\
\hline Female gender & 1.36 & $(0.78-2.37)$ & 1.14 & $(0.63-2.07)$ \\
\hline Manual social class & $3.34^{* *}$ & $(1.69-6.57)$ & 0.98 & $(0.31-3.08)$ \\
\hline GHQ $(2+)$ at age 36 & & & $3.26^{* *}$ & $(1.43-7.45)$ \\
\hline Social class and GHQ interaction & & & $9.57^{* *}$ & $(2.55-35.95)$ \\
\hline
\end{tabular}

${ }^{*} p<0.1$.

$* * p<0.05$. 
Table 3 Odds ratios for psychiatric distress at age 57, with and without adjustment for insomnia symptoms

\begin{tabular}{|c|c|c|c|c|}
\hline & \multicolumn{2}{|c|}{ Model C: Predicting distress } & \multicolumn{2}{|c|}{ Model D: Predicting distress with adjustment for sleep } \\
\hline & OR & $95 \% \mathrm{Cl}$ & OR & $95 \% \mathrm{Cl}$ \\
\hline Female gender (ref: Male) & $1.45^{* *}$ & $(1.01-2.09)$ & 1.24 & $(0.79-1.97)$ \\
\hline Manual social class (ref: Non-manual) & 1.03 & $(0.57-1.87)$ & 0.92 & $(0.47-1.78)$ \\
\hline GHQ (2+) at age 36 (ref: <2) & $2.20^{* *}$ & $(1.55-3.13)$ & $1.69^{* *}$ & $(1.16-2.47)$ \\
\hline Gender and Social class interaction & $2.00^{*}$ & $(0.96-4.14)$ & $2.16^{* *}$ & $(1.05-4.46)$ \\
\hline Social class and GHQ Interaction & $2.19^{* *}$ & $(1.08-4.45)$ & 1.51 & $(0.71-3.20)$ \\
\hline Episodic maintenance class (ref: Healthy) & & & $2.90^{* *}$ & $(1.35-6.25)$ \\
\hline Developing class (ref: Healthy) & & & $4.09^{* *}$ & $(2.36-7.09)$ \\
\hline Chronic mixed class (ref: Healthy) & & & $9.23^{* *}$ & (5.10-16.68) \\
\hline
\end{tabular}

${ }^{*} \mathrm{p}<0.1$.

$* * p<0.05$.

\section{Discussion}

By using 20 years of longitudinal data this paper goes further in demonstrating the inter-relatedness of insomnia and psychiatric distress than is possible with cross-sectional research or longitudinal research that only utilises crosssectional (e.g. baseline) measures of insomnia symptoms. Even after adjustment for baseline psychiatric distress middle-aged women experienced greater odds of developing insomnia symptoms in late middle-age than men, and those who experienced psychiatric distress and socioeconomic disadvantage were more likely than others to be experiencing chronic insomnia symptoms. Psychiatric distress was more likely to recur or still be present 20 years later for those in socioeconomic disadvantage compared to those more affluent, but this association was reduced to non-significance when conditioning on intervening insomnia symptom trajectories. Irrespective of earlier psychiatric distress, women were more likely than men to experience psychiatric symptoms around age 57, though women from a manual social class were particularly disadvantaged in this respect. The greater likelihood of insomnia symptoms developing in middle age among women compared to men explained the overall association between gender and psychiatric distress at age 57, but not the extra disadvantages for women in a manual social class.

The findings concur with previous research showing that psychiatric distress and insomnia symptoms occur more frequently among women and those in socioeconomic disadvantage $[2,3,19]$. Cross-sectional evidence shows that sleep quality can mediate associations between socioeconomic disadvantage and poor mental health $[37,38]$, but these findings show that this can be true over the long-term, up to 20 years, and that gender differences in mental health can also be mediated by sleeping problems. The findings agree with evidence that socioeconomic inequalities in psychiatric distress are at least in part due to symptoms being more persistent among the disadvantaged $[3,39,40]$, and these results show that chronic insomnia symptoms are associated with socioeconomic inequalities in persistent distress. Research showing that more severe insomnia, including problems with both sleep maintenance and initiation, is associated with more severe psychiatric symptoms was also supported [41], and the findings add that this is particularly true for those who experienced long-term problems with both sleep maintenance and initiation.

It is clear from this study that insomnia symptoms and psychiatric distress are strongly associated with each other over the long-term, and that the social patterning of these problems is not independent. The propensity for those in socioeconomic disadvantage to experience persistent or recurring psychiatric distress is strongly associated with the propensity of these individuals for chronic insomnia symptoms, and the propensity for women to experience psychiatric distress in late-middle age is strongly associated with their propensity to develop insomnia symptoms around this time. Poor sleep appears to be characteristic of the type of psychiatric distress which is experienced more often by women and those in socioeconomic disadvantage. This suggests that treatment of insomnia has potential value for alleviating inequalities in psychiatric distress. Inequalities in psychiatric distress might also be helped by tackling the structural or societal constraints that make healthy sleep more difficult for particular groups [42]. The only element of the social patterning of psychiatric distress which was not strongly associated with insomnia symptoms in this study was the particular tendency for women in socioeconomic disadvantage to have high GHQ scores, and so such interventions would be limited in the extent to which they could alleviate this inequality.

Insomnia symptoms are thought to precipitate poor mental health under a diathesis-stress model [43] where 
the stress and opportunities for negative rumination associated with long, wakeful nights facilitate expression of a latent vulnerability to psychopathology [16,41]. Insomnia with short sleep duration can also impact on neurocognitive performance (e.g. attention switching, visual memory, processing speed) [44], and such performance deficits could be distressing to experience. Conversely, anxious, negative rumination may make it more difficult to sleep [16,41]. A diathesis-stress model is also often used to account for socioeconomic inequalities in distress [43], with the accumulation of stressful circumstances and low levels of coping resources associated with socioeconomic disadvantage explaining the higher levels of psychiatric morbidity, rather than differences in underlying vulnerability between social strata $[45,46]$. Socioeconomic disadvantage has additionally been posited as restricting the autonomy required to create ideal conditions for sleep [42]; recommendations such as not taking your problems to bed, for example, could be especially difficulty for those with more problems due to their disadvantaged circumstances, or those in lower status occupations may be more likely to experience disruptions to sleeping patterns from shift work. The inter-play of these processes could easily result in the findings reported here, which suggest a clustering of co-morbid persistent psychiatric distress and chronic insomnia symptoms among those in socioeconomic disadvantage.

As socioeconomic factors partially explain gender differences in psychiatric distress and insomnia symptoms $[11,20,22]$, it is plausible that similar processes partially account for these differences. However, as in previous research, there were still independent effects of gender in this study even after conditioning on social class, and so further explanation is needed beyond gender inequities in the socioeconomic distribution. One hypothesis is that women experience additional stress from domestic and caring roles, over and above roles in employment or elsewhere, but studies have failed to show that this substantially accounts for gender differences in psychiatric distress or insomnia $[22,47,48]$. Female sex may also constitute a biological diathesis, with sex differences having been shown in a number of biological processes connected with depression [49]. This would lead to stronger gender differences where stress levels were also high, such as in a disadvantaged SEP, as was found in this study. A biological process uniquely experienced by women and particularly relevant to the age-group under study here is the menopausal transition, but evidence is mixed as to associations between menopause and depression or insomnia symptoms [50-52], and suggests that links could be accounted for by other life experiences common to women of this age in this cultural context such as the onset of chronic conditions, or children leaving home $[53,54]$. If some of these life experiences were more common amongst people living in socioeconomic disadvantage, then this could also explain the interaction with social class.

The findings support the idea of a positive feedback loop where insomnia and psychiatric distress aggravate each other $[16,41]$. There are nevertheless some limitations to the causal inferences that can be made, considering that the measure of sleep patterning includes indicators of insomnia symptoms at ages 36 and 57 (i.e. concurrent with the measures of psychiatric distress). The insomnia symptoms expressed in the Chronic Mixed or Episodic Maintenance patterns tended to be present at baseline and therefore probably began earlier so it is not clear whether they preceded or were preceded by psychiatric distress or socioeconomic disadvantage. Only with the Developing pattern is there evidence that earlier psychiatric distress predicts the later development of insomnia symptoms. Gender, taken here as an indication of biological sex, probably precedes insomnia symptoms and psychiatric distress (implying causal direction), both because biological sex is usually constant over the lifecourse, and because gender was primarily associated with symptoms that developed post-baseline. Symptomatic sleep patterns did predict distress at age 57 , but were also predicted by distress at age 36 , so the link may be associative rather than causal. Questions about causal direction between sleep and psychiatric distress have been addressed elsewhere, tending to show relationships in both directions $[13-15,17,18,41]$, but the main focus here was on the social patterning. Additionally, considering the five year gaps between measurements, apparently persistent symptom patterns may represent recurring rather than persistent experiences. A further limitation is that people were only observed between ages 36 and 57; date from the USA suggests that the prevalence of sleep disturbance rises between ages 45-59 in men and women, but declines at older ages [55], so the symptoms observed in this cohort may not persist into later life. However, our previous research found higher levels of insomnia symptoms in the older cohort of the Twenty-07 study as they aged from $56-76$ years [23].

\section{Conclusions}

Overall this study demonstrates that the patterning of psychiatric distress and insomnia symptoms by gender and social class are strongly linked. Socioeconomic disadvantage is associated with a tendency to experience both chronic insomnia symptoms and persistent psychiatric distress, whilst women appear to be more likely than men to develop both insomnia symptoms and psychiatric distress in middle age. Recognising the association between inequalities in psychiatric distress and insomnia symptoms may help efforts to alleviate these inequalities. 


\section{Additional file}

\section{Additional file 1: Sensitivity analyses.}

\section{Abbreviations}

SEP: Socioeconomic position; GHQ: General health questionnaire; OR: Odds ratio; Cl: Confidence interval.

\section{Competing interests}

MJG and MB declare that they have no competing interests. CAE declares that he has received payment for other work from Boots UK, Novartis, UCB pharma, and Constable \& Robinson; has received grant funding from the $\mathrm{NIH}$, the Chief Scientist Office in Scotland and Breast Cancer Research; and is a co-founder and shareholder of Sleepio Ltd.

\section{Authors' contributions}

MG designed the study, performed the statistical analysis and drafted the manuscript. CE and MB participated in the design of the study and helped to draft the manuscript. All authors read and approved the final manuscript.

\section{Acknowledgements}

We are grateful to all of the survey participants and staff. The West of Scotland: Twenty-07 Study is funded by the UK Medical Research Council. MG is funded by the UK Medical Research Council. MB is funded by the University of Essex and the UK Economic \& Social Research Council. CE is funded by the University of Oxford. We are grateful to Kate Hunt for comments on earlier versions of the manuscript.

\section{Author details}

${ }^{1} \mathrm{MRC} / \mathrm{CSO}$ Social and Public Health Sciences Unit, University of Glasgow, 4 Lilybank Gardens, Glasgow, G12 8RZ, UK. ${ }^{2}$ Nuffield Department of Clinical Neurosciences/Sleep \& Circadian Neuroscience Institute, University of Oxford, Level 6, West Wing, John Radcliffe Hospital, Oxford, OX3 9DU, UK. ${ }^{3}$ Institute of Social and Economic Research, University of Essex, Colchester, CO4 3SQ, UK

\section{Received: 17 March 2014 Accepted: 20 May 2014}

Published: 25 May 2014

\section{References}

1. Eaton WW, Muntaner C: Socioeconomic stratification and mental disorder. In A handbook for the study of mental health: social contexts, theories, and systems. Edited by Horwitz AV, Scheid TL. Cambridge: Cambridge University Press; 1999:259-283.

2. Fryers $T$, Melzer $D$, Jenkins $R$ : Social inequalities and the common mental disorders: a systematic review of the evidence. Soc Psychiatry Psychiatr Epidemiol 2003, 38(5):229-237.

3. Lorant V, Deliege D, Eaton W, Robert A, Philippot P, Ansseau M: Socioeconomic inequalities in depression: a meta-analysis. Am J Epidemiol 2003, 157(2):98-112.

4. Andersen I, Thielen K, Nygaard E, Diderichsen F: Social inequality in the prevalence of depressive disorders. J Epidemiol Community Health 2009, 63(7):575-581

5. Miech RA, Shanahan MJ: Socioeconomic status and depression over the life course. J Health Soc Behav 2000, 41(2):162-176.

6. Chandola T, Ferrie J, Sacker A, Marmot M: Social inequalities in self reported health in early old age: follow-up of prospective cohort study. Br Med J 2007, 334(7601):990.

7. Bjelland I, Krokstad S, Mykletun A, Dahl AA, Tell GS, Tambs K: Does a higher educational level protect against anxiety and depression? The HUNT study Soc Sci Med 2008, 66(6):1334-1345.

8. Green MJ, Benzeval M: Ageing, social class, and common mental disorders: longitudinal evidence from three cohorts in the West of Scotland. Psychol Med 2011, 41(3):565-574

9. Piccinelli M, Wilkinson G: Gender differences in depression: critical review. Br J Psychiatry 2000, 177(6):486-492.

10. Bijl RV, de Graaf R, Ravelli A, Smit F, Vollebergh WAM: Gender and age-specific first incidence of DSM-III-R psychiatric disorders in the general population: results from the Netherlands Mental Health Survey and Incidence Study (NEMESIS). Soc Psychiatry Psychiatr Epidemiol 2002, 37(8):372-379.
11. Mirowsky J: Age and the gender gap in depression. J Health Soc Behav 1996, 37(4):362-380.

12. McDonough $P$, Strohschein $L$ : Age and the gender gap in distress. Women Health 2003, 17:1-20. September 2003 ed.

13. LeBlanc M, Merette C, Savard J, Ivers H, Baillargeon L, Morin CM: Incidence and risk factors of insomnia in a population-based sample. Sleep 2009, 32(8):1027-1037.

14. Morphy H, Dunn KM, Lewis M, Boardman HF, Croft PR: Epidemiology of insomnia: a longitudinal study in a UK population. Sleep 2007, 30(3):274-280

15. Breslau N, Roth T, Rosenthal L, Andreski P: Sleep disturbance and psychiatric disorders: a longitudinal epidemiological study of young Adults. Biol Psychiatry 1996, 39(6):411-418.

16. Taylor DJ, Durrence HH, Carskadon MA, Morin CM, Stepanski EJ, Lichstein KL: Insomnia as a health risk factor. Behav Sleep Med 2003, 1(4):227-247.

17. Riemann D, Voderholzer U: Primary insomnia: a risk factor to develop depression? J Affect Disord 2003, 76(1-3):255-259.

18. Buysse DJ, Angst J, Gamma A, Ajdacic V, Eich D, Roessler W: Prevalence, course, and comorbidity of insomnia and depression in young adults Sleep 2008, 31(4):473-480.

19. Zhang B, Wing Y-K: Sex differences in insomnia: a meta-analysis. Sleep 2006, 29(1):85-93.

20. Arber S, Bote M, Meadows R: Gender and socio-economic patterning of self-reported sleep problems in Britain. Soc Sci Med 2009, 68(2):281-289.

21. Patel N, Grandner M, Xie D, Branas C, Gooneratne N: "Sleep disparity" in the population: poor sleep quality is strongly associated with poverty and ethnicity. BMC Public Health 2010, 10(1):475.

22. Chen Y-Y, Kawachi I, Subramanian SV, Acevedo-Garcia D, Lee Y-J: Can social factors explain sex differences in insomnia? Findings from a national survey in Taiwan. J Epidemio/ Community Health 2005, 59(6):488-494

23. Green MJ, Espie C, Hunt K, Benzeval M: The longitudinal course of insomnia symptoms: inequalities by sex and occupational class among two different age cohorts followed for 20 years in the West of Scotland. Sleep 2012, 35(6):815-823.

24. Fava M, McCall WV, Krystal A, Wessel T, Rubens R, Caron J, Amato D, Roth T: Eszopiclone co-administered with fluoxetine in patients with insomnia coexisting with major depressive disorder. Biol Psychiatry 2006, 59(11):1052-1060

25. Manber R, Edinger JD, Gress JL, San Pedro-Salcedo MG, Kuo TF, Kalista T: Cognitive behavioral therapy for insomnia enhances depression outcome in patients with comorbid major depressive disorder and insomnia. Sleep 2008, 31(4):489-495.

26. Turek FW: Insomnia and depression: if it looks and walks like a duck. Sleep 2005, 28(11):1362-1363

27. Benzeval M, Der G, Ellaway A, Hunt K, Sweeting H, West P, Macintyre S: Cohort profile: West of Scotland Twenty-07 Study - health in the community. Int J Epidemiol 2009, 38(5):1215-1223.

28. Der G: A comparison of the West of Scotland Twenty-07 Study sample and the 1991 census SARs. In MRC Medical Sociology Unit Working Paper. Glasgow: MRC Medical Sociology Unit; 1998.

29. Goldberg D, Williams P: A User's Guide to the General Health Questionnaire Windsor: NFER-Nelson; 1988.

30. Office of Population Censuses and Surveys: Classification of Occupations. London: HMSO; 1980.

31. Asparouhov T, Muthén BO: Auxiliary variables in mixture modeling: a 3-step approach using Mplus. [http://www.statmodel.com/download/ 3stepOct28.pdf].

32. Muthén LK, Muthén BO: Mplus User's Guide. 7th edition. Los Angeles, CA: Muthén \& Muthén; 1998. 2012.

33. Seaman S, Benzeval M: The West of Scotland Twenty-07 study: inverse probability weights for Wave 5 . In Working Paper. Glasgow: MRC/CSO Social and Public Health Sciences Unit; 2011

34. Asparouhov T, Muthén B: Multiple Imputation with Mplus. Technical Report (version 2). ; 2010. http://www.statmodel.com/download/Imputations7.pdf.

35. Clarke P, Hardy R: Methods for handling missing data. In Epidemiological Methods in Life Course Research. Edited by Pickles A, Maughan B, Wadsworth M. Oxford: Oxford University Press; 2007:157-180.

36. Schafer JL: Analysis of incomplete multivariate data. London: Chapman \& Hall; 1997.

37. Moore PJ, Adler NE, Williams DR, Jackson JS: Socioeconomic status and health: the role of sleep. Psychosom Med 2002, 64(2):337-344. 
38. Sekine M, Chandola T, Martikainen P, McGeoghegan D, Marmot M, Kagamimori S: Explaining social inequalities in health by sleep: the Japanese civil servants study. J Public Health 2006, 28(1):63-70.

39. Skapinakis P, Weich S, Lewis G, Singleton N, Araya R: Socio-economic position and common mental disorders: longitudinal study in the general population in the UK. Br J Psychiatry 2006, 189(2):109-117.

40. Green MJ, Benzeval M: The development of socioeconomic inequalities in anxiety and depression symptoms over the lifecourse. Soc Psychiatry Psychiatr Epidemiol 2013, 48(12):1951-1961.

41. Taylor DJ, Lichstein KL, Durrence H, Reidel BW, Bush AJ: Epidemiology of insomnia, depression, and anxiety. Sleep 2005, 28(11):1457-1464

42. Hale L, Hale B: Treat the source not the symptoms: why thinking about sleep informs the social determinants of health. Health Educ Res 2010, 25(3):395-400

43. Monroe SM, Simons AD: Diathesis-stress theories in the context of life stress research: implications for the depressive disorders. Psychol Bull 1991, 110(3):406-425.

44. Fernandez-Mendoza J, Calhoun S, Bixler E, Pejovic S, Karataraki M, Liao D, Vela-Bueno A, Ramos-Platon M, Sauder K, Vgontzas A: Insomnia with objective short sleep duration is associated with deficits in neuropsychological performance: a general population study. Sleep 2010, 33(4):459-465.

45. Turner RJ, Wheaton B, Lloyd DA: The epidemiology of social stress. Am Sociol Rev 1995, 60(1):104-125.

46. Turner RJ, Lloyd DA: The stress process and the social distribution of depression. J Health Soc Behav 1999, 40(4):374-404.

47. Weich S, Sloggett A, Lewis G: Social roles and the gender difference in rates of the common mental disorders in Britain: a 7-year, population-based cohort study. Psychol Med 2001, 31(6):1055-1064.

48. Weich S, Sloggett A, Lewis G: Social roles and gender difference in the prevalence of common mental disorders. Br J Psychiatry 1998, 173(6):489-493.

49. Halbreich $U$, Lumley LA: The multiple interactional biological processes that might lead to depression and gender differences in its appearance. J Affect Disord 1993, 29(2-3):159-173.

50. Avis NE, Brambilla D, McKinlay SM, Vass K: A longitudinal analysis of the association between menopause and depression results from the Massachusetts women's health study. Ann Epidemio/ 1994, 4(3):214-220.

51. Owens JF, Matthews KA: Sleep disturbance in healthy middle-aged women. Maturitas 1998, 30(1):41-50.

52. Freeman EW, Sammel MD, Liu L, Gracia CR, Nelson DB, Hollander L: Hormones and menopausal status as predictors of depression in women in transition to menopause. Arch Gen Psychiatry 2004, 61(1):62-70.

53. Mitchell ES, Woods NF: Symptom experiences of midlife women: observations from the Seattle midlife women's health study. Maturitas 1996, 25(1):1-10.

54. McKinlay JB, McKinlay SM, Brambilla D: The relative contributions of endocrine changes and social circumstances to depression in mid-aged women. J Health Soc Behav 1987, 28(4):345-363.

55. Grandner MA, Martin JL, Patel NP, Jackson NJ, Gehrman PR, Pien G, Perlis ML, Xie D, Sha D, Weaver T, Gooneratne NS: Age and sleep disturbances among American men and women: data from the U.S. behavioral risk factor surveillance system. Sleep 2012, 35(3):395-406.

doi:10.1186/1471-244X-14-152

Cite this article as: Green et al:: Social class and gender patterning of insomnia symptoms and psychiatric distress: a 20-year prospective cohort study. BMC Psychiatry 2014 14:152.

\section{Submit your next manuscript to BioMed Central and take full advantage of:}

- Convenient online submission

- Thorough peer review

- No space constraints or color figure charges

- Immediate publication on acceptance

- Inclusion in PubMed, CAS, Scopus and Google Scholar

- Research which is freely available for redistribution

Submit your manuscript at www.biomedcentral.com/submit 